\title{
Estratégias de preparação física e prevalência de lesões em atletas de rodeio profissional
}

\section{Physical fitness strategies and prevalence of injuries in professional rodeo athletes}

Franciele Martins de Andrade ${ }^{1}$, Leonardo Ferreira Rocha ${ }^{1}$, Vera Maria Cury Salemi², Douglas Pinheiro Miranda ${ }^{1,2}$

1- Laboratório de Avaliação Física e Fisiologia do Exercício (LAFFEX), Curso de Educação Física, Centro Universitário da Fundação Educacional de Barretos, Barretos, SP, Brasil.

2- Programa de Doutorado em Cardiologia, Instituto do Coração, Faculdade de Medicina da Universidade de São Paulo, São Paulo, SP, Brasil.

\begin{abstract}
Resumo
O rodeio é um esporte radical que exige do atleta técnica, força, foco mental e uma preparação física antes da temporada. Este esporte tem como consequência grande risco de lesões, porém no Brasil não estão elucidadas quais são as lesões mais frequentes entre os peões nem quais estratégias são utilizadas como preparação física. Este estudo tem como objetivo investigar as estratégias de preparação física bem como a prevalência de lesões entre atletas profissionais do rodeio nas modalidades montaria em touros e montarias em cavalos estilo cutiano. Durante a $60^{\mathrm{a}}$ Festa do Peão de Boiadeiro de Barretos, foram entrevistados 50 atletas das modalidades montaria em touros e montaria em cavalos estilo cutiano. Foi constatado que $66 \%$ dos atletas realizam algum tipo de preparação física, porém apenas $36 \%$ afirmaram ter a orientação de um profissional de educação física. A estratégia mais utilizada para preparação física é a musculação e a corrida. Quanto à incidência de lesões, 49 atletas afirmaram já ter sofrido algum tipo de lesão; entre as mais citadas estão fraturas, luxações e distensões musculares. Conclui-se que os atletas de rodeio conhecem a importância do exercício físico durante sua preparação profissional e que a musculação e a corrida são as atividades mais utilizadas por esses atletas. Concluímos também que a montaria é um esporte com alto índice de lesões, sobretudo fraturas, luxação e distensões musculares.
\end{abstract}

Palavras-chave: atleta; lesão; treinamento.

\begin{abstract}
Rodeo is a radical sport which requires the athlete's technique, strength, mental focus and a physical preparation before the season. This sport results in a high risk of injury, however in Brazil it is not clear which are the most frequent injuries among pedestrians, nor what strategies are used as physical preparation. This study aims to investigate the strategies of physical preparation as well as the prevalence of injuries among professional athletes of the rodeo modalities in bulls and mounts in Cutiano style horses. During the 60th Celebration of the Pawn of Boiadeiro of Barretos, 50 athletes of the modalities were mounted in bulls and mount in horses Cutiano style. It was found that $66 \%$ of the athletes performed some kind of physical preparation, but only $36 \%$ affirmed that they had the guidance of a Physical Education professional. The most used strategy for physical preparation is bodybuilding and running, as for the incidence of injuries, 49 athletes have already suffered some type of injury, among the most cited are fractures, dislocations
\end{abstract}

Autor correspondente: Douglas Pinheiro Miranda Av. Prof Roberto Frade Monte, 386 Barretos, SP, Brasil CEP:14783-226 e-mail: douglas@pinheiromiranda.com

Recebido em: 17/09/2018

Aceito para publicação em: 26/09/2019

https://doi.org/10.4322/1980-0029.042020 
and muscle strains. It was possible to conclude that the rodeo athletes know the importance of physical exercise during their professional preparation, and that bodybuilding and running are the activities most used by these athletes. Besides that, it was also concluded that the mount is a sport with high lesion index, especially fractures, dislocation and muscle strains.

Keywords: Athlete; Injury; Training.

\section{Introdução}

O rodeio teve sua origem nos Estados Unidos no ano de 1870, após alguns homens que levavam grandes rebanhos para os entroncamentos ferroviários começarem a apostar corridas em seus cavalos e montar em touros bravos como forma de diversão (Duarte, 2003).

No Brasil o rodeio também tem sua origem na diversão dos peões das fazendas que montavam nos touros aos finais de semana. Na cidade de Barretos, interior de São Paulo, em meados de 1955 o município tinha toda sua economia voltada à agropecuária. Havia instalado na cidade um frigorifico muito conhecido. Assim, o fluxo de vaqueiros que levavam as boiadas para a cidade era enorme (Independentes, 2012).

No ano de 2001 o ex-presidente Fernando Henrique Cardoso sancionou a lei federal $\mathrm{n}^{\mathrm{o}} 10.220$, de 11 de abril, na qual o peão passou a ser considerado um atleta profissional. No ano seguinte o rodeio tornou-se uma atividade esportiva. Essas leis fizeram com que o competidor ganhasse alguns benefícios, como a obrigatoriedade de um seguro de vida em cada competição que ele participar (Brasil, 2002).

Segundo Costa (2006), o Brasil é considerado o segundo país com maior organização de regras no rodeio, perdendo apenas para os Estados Unidos. Essa situação é claramente percebida devido ao grande número de eventos principalmente nas regiões Sudeste e Centro-Oeste, gerando muito lucro para as cidades que sediam este tipo de competição.

O rodeio brasileiro é dividido nas seguintes modalidades: montaria em touros, montaria em cavalos e provas cronometradas. Nesta pesquisa entrevistamos competidores da montaria em touros e atletas da montaria em cavalos estilo cutiano.

O objetivo do atleta da montaria em touros é permanecer no animal durante 8 segundos. Para isso ele se senta no touro mantendo os quadris flexionados, segura uma das mãos na corda americana que é presa ao corpo do marruco (pegada supinada), faz uma adução da coxa e flexão dos joelhos. Quando o peão percebe que está corretamente posicionado, ele pede para abrir a porteira e então o touro começa a pular, girar e correr (dependendo de cada animal) e o atleta, na tentativa de prever o movimento do touro, olha para a cabeça do animal. Nesse momento o competidor deve manter a musculatura do abdômen em uma contração isométrica, continuar realizando uma adução da coxa e flexão dos joelhos. A outra mão que fica livre no ar como forma de equilíbrio e correção da posição do corpo fica posicionada em um ângulo de aproximadamente $90^{\circ}$, porém depende muito da técnica de cada competidor e do movimento que o touro realiza.

O mesmo acontece na montaria em cavalos estilo cutiano, porém nesta modalidade os atletas realizam, durante toda a montaria, flexão e extensão dos joelhos, mantendo as esporas na altura do pescoço do equino, exigindo, assim, um grande esforço dos joelhos.

É possível notar que durante 8 segundos as maiores ações musculares são: flexão e extensão do cotovelo, quadril, tronco e joelhos; isometria do abdômen, estabilização do ombro e adução da coxa.

Este esporte considerado radical (Costa et al., 2007) exige do atleta uma combinação entre o foco mental, capacidade técnica, além de uma preparação física pré-temporada que ajuda o peão em seu desempenho, além de diminuir a incidência de lesões (Barrett, 2006).

Uma pesquisa realizada no Canadá durante os anos de 1995 a 1999 constatou que quase a metade das lesões em competidores da modalidade montaria em touros são nos joelhos e ombros. Outras lesões não tão comuns, porém mais graves ocorrem por ferimentos no rosto e pescoço (Butterwick \& Meeuwisse, 2003).

Entretanto, no Brasil não estão totalmente elucidadas quais são as lesões mais comuns entre atletas de rodeio bem como as estratégias de preparação física adotadas para esta modalidade.

Este artigo tem como objetivo investigar as estratégias de preparação física bem como a incidência de lesões entre atletas profissionais do rodeio nas modalidades montaria em touros 
e montarias em cavalos estilo cutiano, podendo assim conseguir uma maior divulgação do esporte, além de aprimorar os métodos de preparação física para atletas do rodeio.

\section{Materiais e métodos}

Participaram do estudo 50 atletas com idade entre 18 e 37 anos $(26,8 \pm 5,2$ anos), sendo 12 da montaria em cavalos estilo cutiano e 38 da montaria em touros, com tempo de prática na modalidade de $8,6 \pm 4,4$ anos. Todos assinaram o termo de consentimento livre e esclarecido (TCLE) e participaram voluntariamente desta pesquisa, que teve seu projeto previamente aprovado pelo comitê de ética em pesquisa do Centro Universitário da Fundação Educacional de Barretos (CAAE: 31345314.8.0000.5433).

A coleta de dados ocorreu durante a $60^{\circ}$ Festa do Peão de Boiadeiro de Barretos que aconteceu de 20 a 30 de agosto de 2015, a qual foi dividida em quatro etapas de rodeio diferentes, sendo elas: final PBR Brasil 2015 (Profissional Bull Riders), final da Liga Nacional de Rodeio (LNR), primeira etapa do PBR Brasil 2016 e rodeio internacional de Barretos.
Os dados foram coletados por meio de entrevista, na qual os atletas foram questionados de acordo com o que segue na Tabela 1.

Para o tratamento estatístico foi utilizado o programa Microsoft Excel Office 2010, de modo que os dados pudessem ser apresentados em valores absolutos, percentuais, média e desvio padrão.

\section{Resultados}

Após análises dos resultados gerais, observamos que os atletas participam de 27,8 $\pm 12,11$ competições por ano. Dos 50 entrevistados, 33 atletas fazem preparação física, ou seja, $66 \%$, com uma frequência semanal de 2,6 $\pm 1,8$ dias. Entretanto apenas $18(36 \%)$ alegaram possuir orientação de um profissional de educação física.

$\mathrm{Na}$ Figura 1 destacamos quais modalidades são utilizadas como estratégia de preparação física dos atletas.

O rodeio é considerado um esporte muito perigoso e com grande risco de lesões nos praticantes da modalidade. As lesões mais relatadas pelos atletas deste estudo foram: fraturas, distensões musculares, ruptura ligamentar e entorses. A Figura 2 mostra a prevalência de lesões.

Tabela 1. Questões utilizadas na entrevista.

1) Há quanto tempo pratica esta modalidade?
2) Qual tipo de prova participa (modalidade)?
3) Em média, de quantas competições você participa por ano?
4) Tem algum outro trabalho remunerado fora das competições de rodeio?
5) Faz alguma preparação física? Qual? Quantas vezes por semana?
6) Esta preparação é orientada por um profissional de educação física?
7) Possui algum histórico de lesão? Se a resposta for sim, quais foram essas lesões?

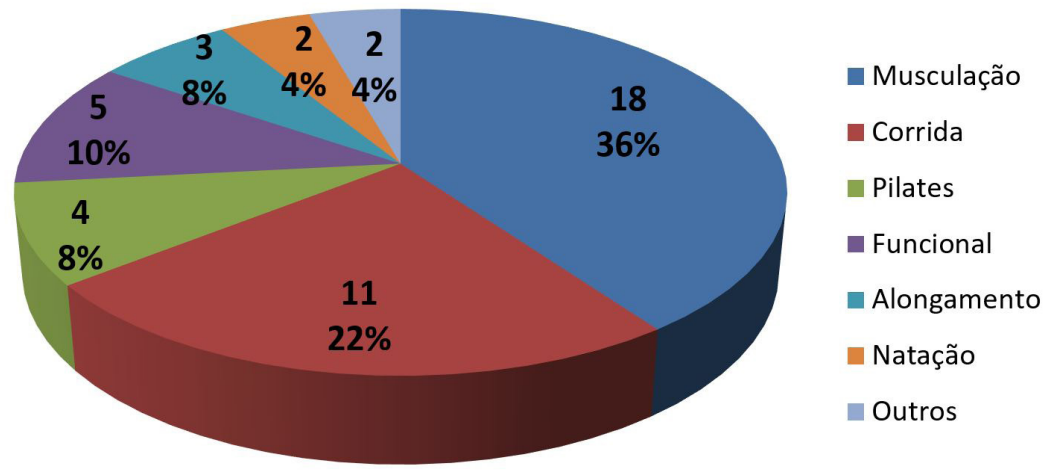

Figura 1. Modalidades utilizadas na preparação física 


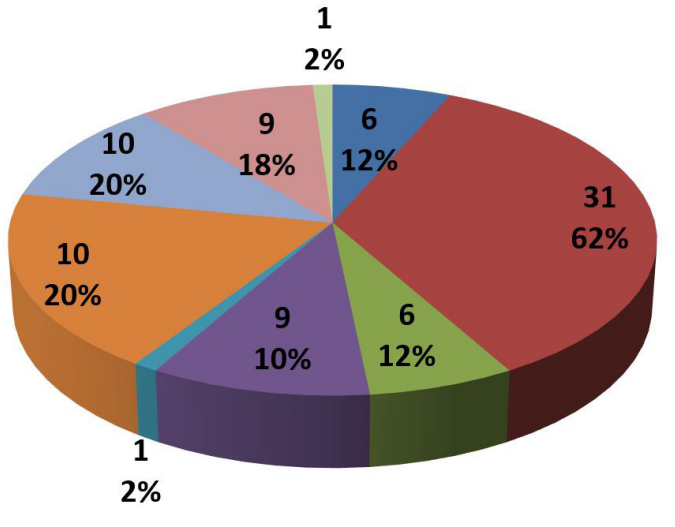

Nenhuma

- Fraturas

Corte na face

nuptura ligamentar

Rompimento do bícpes

Distensão Muscular

Luxação

Entorse

Figura 2. Lesões de atletas do rodeio em touros e cavalos

É importante destacar que dos 17 atletas que já tiveram distensão muscular, 16 são da modalidade montaria em touros, sete dos nove atletas que tiveram ruptura ligamentar também são da montaria em touros e seis que alegaram não ter nenhum histórico de lesão, dois também são desta modalidade.

\section{Discussão}

De acordo com os resultados do presente estudo, dos 50 atletas entrevistados, 33 (66\%), ou seja, mais da metade relatou incluir programas de exercício físico na preparação profissional com frequência semanal de no mínimo dois dias. No entanto apenas $18(36 \%)$ possuem acompanhamento de um profissional de educação física. Segundo Branicio (2012), os atletas de rodeio conhecem a importância da preparação física, sobretudo com a orientação de um profissional. Entretanto, tendo em vista que dificilmente um atleta de rodeio tem a oportunidade de desfrutar de algum tipo de auxílio financeiro (patrocínio), alguns acabam não investindo na preparação física, pois essa atividade pode aumentar suas despesas.

Ainda em relação à preparação física, também gostaríamos de destacar que entre os 33 atletas que praticam algum tipo de exercício físico durante a preparação profissional, 54,5\% utilizam o treinamento resistido e $33,3 \%$ são adeptos à corrida. Desta forma, podemos observar que os tradicionais métodos de treinamento (treinamento resistido e corrida) são os mais utilizados entre os atletas de rodeio.
Analisando as características especificas da modalidade, durante os oito segundos, os atletas de rodeio passam por esforços intermitentes de alta intensidade. Desta forma, considerando essa característica, a literatura mostra que tanto a corrida quando o treinamento resistido podem ser essenciais para a preparação profissional dos atletas.

Para Thoden (1991), possuir um bom condicionamento cardiorrespiratório pode aumentar a capacidade do músculo recuperar após um esforço intermitente de alta intensidade, ou seja, a participação do metabolismo aeróbio durante a montaria contribui para uma maior regeneração de fosfocreatina, fonte energética diretamente ligada à esforços intermitentes de alta intensidade. No entanto o treinamento deve se concentrar na força máxima, força específica, potência e velocidade durante períodos longe da concorrência. Sabe-se que um grande grau de melhoria de força virá não apenas de adaptações celulares, mas também de adaptações neurais, tais como aumento da ativação de unidades motoras e coordenação de motores primários, sinergistas e antagonistas (Barrett, 2006).

Em um artigo publicado recentemente, Segura \& Sena (2016) também investigou as estratégias de preparação física utilizadas pelos atletas de rodeio e quais eram as lesões mais comuns encontradas nessa modalidade. O presente estudo avaliou 44 atletas, com idade entre 20 e 37 anos $(27,11 \pm 4,35$ anos). Foi constatado que a maioria dos entrevistados $(97,72 \%)$ realizavam algum tipo de exercício físico e, assim como o presente estudo, a musculação $(46,5 \%)$ e a corrida $(65,1 \%)$, 
juntamente com o alongamento $(55,8 \%)$, foram as atividades que os atletas mais utilizam durante a preparação física. Em relação a incidência de lesões dos atletas, o autor dividiu a investigação em dois momentos. No primeiro momento, ele questionou os atletas sobre a ocorrência de lesões que tiveram durante a montaria (8 segundos). Já no segundo momento foram investigadas as lesões que eles tiveram no momento da queda. Durante o tempo em que o atleta tentou se manter em cima do touro, $88,63 \%$ deles sofreram algum tipo de lesão e as lesões mais ocorrentes foram exatamente as evidenciadas pelo presente estudo, sendo fraturas $(41,66 \%)$, distensões musculares $(31,66 \%)$, luxações $(19,16 \%)$. Já durante a queda, $72,72 \%$ dos atletas relataram ter sofrido alguma lesão ao cair de cima do touro.

Em outro estudo investigando a ocorrência de lesões em atletas que participaram da Festa do Peão de Barretos (2004), Bergamaschi et al. (2006) analisou a ocorrência de lesões de 208 atletas, que foi estabelecida de acordo com o número de atendimentos médicos realizados durante a competição. Segundo o autor, durante os 11 dias de competição ocorreram 25 atendimentos no departamento médico local, correspondendo a aproximadamente um lesionado a cada 30 montarias. A lesão mais prevalente foi contusão em $56 \%$ dos lesionados e os locais de acometimento da lesão mais frequentes foram membros superiores em $36 \%$ e membros inferiores em $24 \%$ dos lesionados.

\section{Conclusão}

Diante dos resultados é possível concluir que os atletas de rodeio conhecem a importância do exercício físico durante sua preparação profissional, e que a musculação e a corrida são as atividades mais utilizadas por esses atletas.

Concluímos também que a montaria é um esporte com alto índice de lesões, sobretudo fraturas, luxação e distensões musculares.

\section{Referências}

BARRETT, R. M. (2006). Bull riding: analysis and application of a specific strength and conditioning program. Strength and Conditioning Journal, 28, 10-19.
BERGAMASCHI, J. P., MATSUDO, S. M., \& MATSUDO, V. K. R. (2006). Relação da força de membros superiores e nível de atividade física com a prevalência de lesão e o desempenho de participantes de rodeio competitivo. Revista Brasileira Ciência e Movimento, 14(1), 53-58.

BRANICIO, C. R. (2012). A importância da preparação física para atletas de rodeio de montaria em touros (Trabalho de conclusão de curso). Universidade de Brasília, Polo Barretos.

BRASIL. (2002, 18 de julho). Dispõe sobre a promoção e a fiscalização da defesa sanitário animal quando da realização de rodeio e dá outras providências (Lei Federal no 10.519 de 17 de Julho 2002). Diário Oficial [da] República Federativa do Brasil, Brasília.

BUTTERWICK, D. J., \& MEEUWISSE, W. H. (2003). Bull riding injuries in professional rodeo. The Physician and Sportsmedicine, 31(6), 37-41.

COSTA, S. P. (2006). Esporte e Paixão: o processo de regulamentação os rodeios no Brasil. Revista Movimento, 9(2), 71-87.

COSTA, V. L. M., MARINHO, A., \& PASSOS, K. C. M. (2007). Esportes de Aventura e esportes radicais: propondo conceitos. Revista Motriz. In Anais do V Congresso Internacional de Educação Física e Motricidade Humana e XI Simpósio Paulista 57 de Educação Física (Vol. 13). Rio Claro: UNESP.

DUARTE, O. (2003). Rodeio, história dos esportes (4. ed. pp. 435-436). São Paulo: Editora SENAC. São Paulo.

INDEPENDENTES. (2012). Festa do peão. Barretos: Independentes. Recuperado em 28 de março de 2015, de http://www.independentes. com.br/festadopeao/historiarodeio

SEGURA, D. C. A., \& SENA, J. S. (2016). Lesões em atletas de montaria em touro e prevenção por meio de preparo físico. Saúde e Pesquisa, 9(1), 43-51.

THODEN, J. S. (1991). Testing aerobic power: physiological testing of the high-performance athlete. Champaign: Human Kinetics. 